\title{
RECIPROCAL SPACE ANALYSIS OF THE INITIAL STAGES OF STRAIN RELAXATION IN SiGe EPILAYERS
}

\author{
i S. R. LEE and J. A. FLORO \\ Sandia National Laboratories, Albuquerque, NM 87185-1056, srlee@sandia.gov
}

\begin{abstract}
Metastable SiGe films were grown by MBE on $\mathrm{Si}(001)$ substrates and annealed to promote varying degrees of partial relaxation. X-ray diffraction reciprocal-space analysis was then used to monitor the structural evolution of the displacement fields of the dislocation array with increasing misfit density. The diffuse-x-ray-scattering patterns of the dislocated heterolayers were compared with lineal-misfit densities determined by defect etching, leading to the develop a geometric model which provides a framework for understanding the early-stage evolution of the displacement fields of the dislocation array, and which also explicitly links diffuse x-ray intensity to misfit density. At low misfit density, the diffuse intensity arises from two-dimensional displacement fields associated with single-nonoverlapping dislocations. As misfit density increases, the displacement fields of individual dislocations increasingly overlap producing three-dimensional displacements. The evolving diffuse intensity reflects the transition from 2-D to 3-D displacement fields. Finally, it is demonstrated that the diffuse $x$-ray intensity of the strained epilayer can be used to accurately measure lineal misfit-dislocation densities from 400 to 20,000 lines $/ \mathrm{cm}$.
\end{abstract}

\section{INTRODUCTION}

Defects in crystals can produce diffuse $x$-ray scattering intensity in regions of reciprocal space adjacent to the (hkl) Bragg peaks of the crystal. The shape and intensity of the diffuse scattering as measured in reciprocal space depends on the real-space displacement field of the defects, the microstructural arrangement of the defects, and the density of the defects. We are making detailed reciprocal space measurements of the diffuse scattering produced by $60^{\circ}$-degree misfit-dislocation arrays in strained SiGe heterolayers in order to explore this linkage between the reciprocal-space structure of the diffuse scattering and the real-space structure of the defectivity.

Three intertwined themes are of particular interest to us at present. First, can measurement of the diffuse intensity of the dislocation array at different points in its structural evolution provide any interesting insights into the strain relaxation process? Or alternatively stated, how are diffraction measurements of this evolving structure most properly interpreted? Second, can the diffuse intensity produced by an orthogonal-array of misfit dislocations be used to accurately quantify misfit-dislocation density in the low-density regime where the main Bragg peak still appears as fully strained? Finally, what is the detailed reciprocal-space structure of a low-density array of $60^{\circ}$-misfit dislocations in a strained heterolayer? Here, we address these questions by using $\mathrm{x}$-ray diffraction and defect etching techniques to systematically examine a series of partially relaxed SiGe heterolayers with lineal misfit densities from 0 to $\approx 20,000$ lines $/ \mathrm{cm}$.

\section{EXPERIMENTAL DETAILS}

Metastable SiGe layers were grown by MBE on Shiraki-cleaned Si (001) substrates. The depositions consisted of a $1200 \AA$-thick Si buffer layer followed by the SiGe heterolayer, which was grown at a rate of $\approx 1 \AA / \mathrm{s}$ at $450-550{ }^{\circ} \mathrm{C}$ in order to promote metastability. Heterolayers with a nominal composition of $\mathrm{Si}_{78} \mathrm{Ge}_{22}$, and of two thicknesses: $748 \AA$ and $1208 \AA$, were studied. Wafers were saw diced into samples and solvent cleaned prior to annealing. Samples were mounted face-to-face in a quartz boat and annealed in a quartz tube furnace under flowing dry $\mathrm{N}_{2}$ in order to introduce dislocations. Annealing times (15-105 minutes) and temperatures $\left(450-875^{\circ} \mathrm{C}\right)$ were varied as needed to produce widely varying degrees of dislocation density.

Reciprocal space analysis of the as-grown and dislocated SiGe heterolayers was carried out using a double-crystal, $x$-ray diffractometer which has been modified by the addition of a position-sensitive $\mathrm{x}$-ray detector (PSD) $[1,2]$. The PSD simultaneously measures the total 
scattering angle of diffracted $x$-rays emerging from the sample over a wide range of $2 \theta$ angles. Parallel analysis of multiple scattering angles allows one to map reciprocal space more than 100 times faster than can be done using a traditional, serially-scanned, triple-axis diffractometer.

This efficiency offers unique views of reciprocal space -- especially so for weak, diffuse diffraction. Even though the detection scheme used here is very efficient, scan times of $\approx 12$ hours were required in order to thoroughly examine the diffuse $\mathrm{x}$-ray scattering for the earliest, very low-dislocation-density stages of relaxation. Thus, the present results are almost inaccessible to a conventional triple-axis system using a sealed-tube or rotating anode $\mathrm{x}$-ray source because of the prohibitively long time required to serially map the same region of reciprocal space. There are tradeoffs when using a PSD-based mapping scheme: these are decreased resolution and dynamic range relative to that of a triple-axis system [3].

Following $x$-ray analysis, the defectivity of the samples was revealed using a Schimmel etch: $4\left(0.3 \mathrm{M} \mathrm{CrO}_{3}\right): 5(\mathrm{HF})$. The etchant was diluted to $2(\mathrm{Schimmel}): 3\left(\mathrm{H}_{2} \mathrm{O}\right)$ to reduce the etch rate. Samples were dipped and examined in intervals to obtain optimum contrast for Nomarskidifferential-interference-contrast optical microscopy of the dislocation lines.

\section{DISPLACEMENT FIELDS AND THE DISLOCATION ARRAY GEOMETRY}

Both the evolution of reciprocal space maps with dislocation density, and the quantitative relationship between lineal-misfit density and integrated-diffuse-scattering intensity, are best discussed within the framework of a rather simple geometric model of the orthogonal-dislocation array. A schematic of this model is shown in Fig. 1. It is assumed that the dislocation density is approximately equal along both line directions, and that the array can be approximated by a uniform grid of dislocation lines separated by an average spacing, $d$. A final assumption is that the displacement field extends out laterally from the dislocation line a finite distance, $w / 2$, along the heterointerface. Within this lateral distance, the displacement field extends vertically throughout the epilayer thickness, t. This artificially partitions the epilayer into two domains -distorted domains within $\sim w / 2$ of the dislocation line, and domains outside of this boundary which are considered to be nearly perfect crystalline material.

Given this geometric framework it is easy to derive an equation for the total volume fraction of epitaxial material that lies within distorted domains, $V_{t}$, in terms of the dislocation density, $1 / \mathrm{d}$. This equation appears in Fig. 1. Also defined in Fig. 1 is $\mathrm{V}_{\mathfrak{i}}$, the volume fraction of epitaxial material within overlapping distorted domains formed at intersections in the array. These volume fractions are plotted in terms of the dimensionless parameter, w/d, in Fig. 2. The relative fraction of displaced material within intersections, given by the ratio $V_{i} / V_{t}$, is also shown in Fig. 2 .

Note that $\mathrm{V}_{\mathrm{i}} / \mathrm{V}_{\mathrm{t}}<0.1$ for all values of $\mathrm{w} / \mathrm{d}<0.2$. Thus, during the earliest stages of formation

Total displacement field volume fraction: $V_{t}=\frac{w}{d}\left(2-\frac{w}{d}\right)$

Volume fraction in intersections: $V_{1}=\left(\frac{w}{d}\right)^{2}$

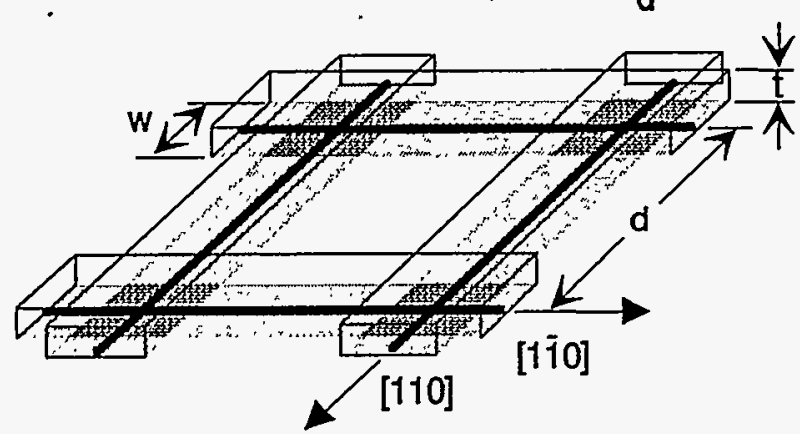

Figure 1. A geometric model of a 60 -degree misfit-dislocation array which partitions the epilayer into distorted and perfect domains.

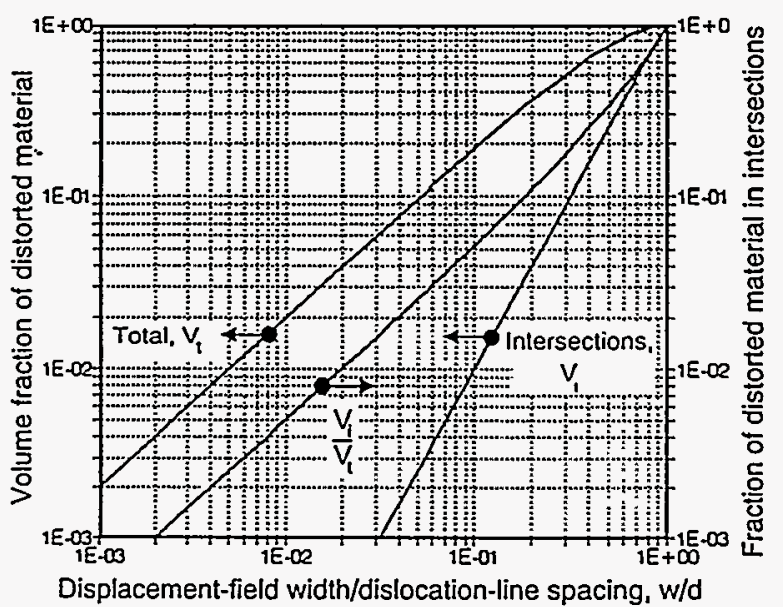

Figure 2. Total and intersectional volume fractions of distorted material in the epilayer versus the relative dislocation density, w/d. 
of the misfit array, the largely nonoverlapping displacement fields near the heterointerface are two-dimensional in character and are characteristic of single dislocations. As the dislocation density rises, there is a transitional regime given by $0.2<w / d<1$, where $V_{i} / V_{t}$ rises rapidly with dislocation density from 0.1 to 1 . As $V_{i} / V_{t}$ approaches unity, the epilayer is essentially comprised entirely of "intersections". Assuming linear superposition of the displacement fields of the individual dislocations making up the array, the displacement fields in intersecting domains must be three-dimensional, rather than two-dimensional, in character. Consequently, this transitional regime is one from two-dimensional to three-dimensional displacement fields.

In sum, the displacement fields making up the dislocation array are essentially nonoverlapping, two-dimensional, and independent for $\mathrm{w} / \mathrm{d}<0.2$, are transitional for $0.2<\mathrm{w} / \mathrm{d}<1$, and are fully overlapping and three-dimensional for $w / d>1$. These characteristics of the displacement fields are apparent in the reciprocal space maps of a partially relaxed heterolayer and are essential to proper understanding and interpretation of the maps.

\section{EVOLUTION OF THE RECIPROCAL SPACE MAPS WITH DISLOCATION DENSITY}

Figures 3 and 4 show (113) reciprocal space maps of $748 \AA$-thick $\mathrm{Si}_{78} \mathrm{Ge}_{22}$ heterolayers for a series of annealing conditions. The maps are cropped to omit the Si substrate peak region. Fig. 3 (a) shows the map of a dislocation-free reference specimen. The fully strained $\mathrm{Si}_{78} \mathrm{Ge}_{22}$ (113) Bragg peak lies at $\left(\mathrm{K}[\mathrm{hh} 0]=1.636 \AA^{-1}, \mathrm{~K}[001]=3.419 \AA^{-1}\right)$. Looking along the [001] direction, one can see the thickness fringes of the essentially perfect epilayer (look along the page at neargrazing incidence to provide the clearest view of the fringes). The diffuse intensity on either side of the SiGe Bragg peak is background intensity that results from wavelength dispersion in the $\mathrm{x}$ ray beam produced by our single-reflection monochromator. The two long streaks in the map, in a direction just off parallel to [001], are detector artifacts, and are analogous to the so-called "analyzer streak" found in triple-axis measurements [3]. In order to accommodate the limited dynamic range of our PSD, the $\mathrm{x}$-ray intensity must be reduced when scanning through the highreflectivity substrate peak [3]. This explains the low intensity observed for the analyzer streak of the Si substrate just to the right of the SiGe Bragg peak. Note that these streaks mark the orientation of the Ewald sphere.

Now that we are oriented, consider the evolution of the diffraction pattern of the epilayer with increasing dislocation density. Fig. 3(b) shows the map of a sample annealed to produce a lineal misfit density of $1400 / \mathrm{cm}$. The introduction of dislocations produces two diffuse-intensity

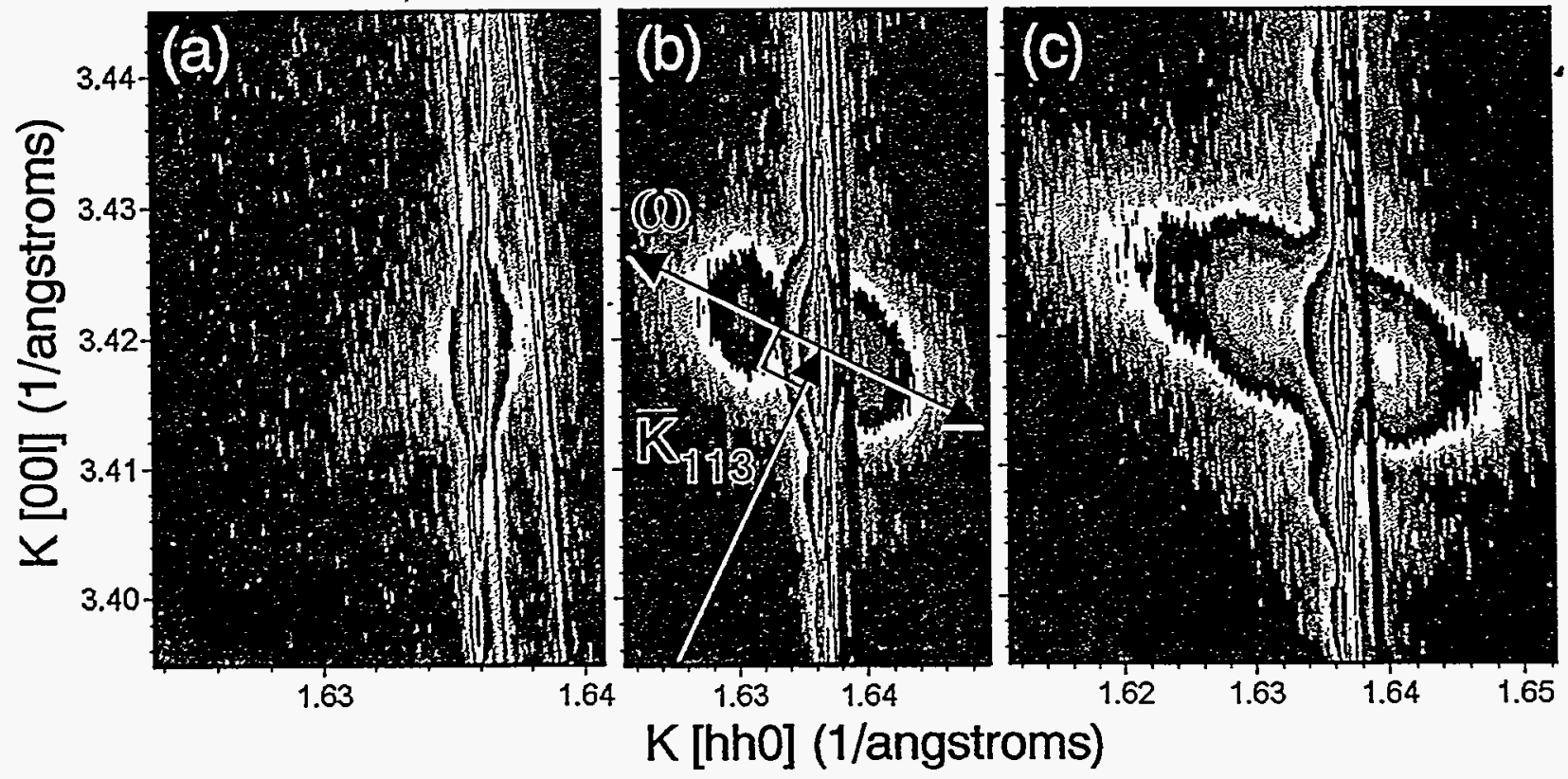

Figure 3. (113) reciprocal space maps of $748 \AA$-thick $\mathrm{Si}_{78} \mathrm{Ge}_{22}$ : (a) as-grown, (b) annealed at 450 ${ }^{\circ} \mathrm{C}$ for 1 hour, and (c) annealed at $700{ }^{\circ} \mathrm{C}$ for 20 minutes. 
features. The first is the pair of large, diffuse "disks" of intensity on either side of the [001] rod. These disks are similar to the so-called "halos" and "lobes" faintly observed by Kidd, et al. during their heroic efforts to map the (004) diffuse X-ray scattering of dislocated InGaAs heterolayers using a conventional triple-axis system [4]. The second diffuse-intensity feature observed in Fig. 3 (b) is the very narrow, asymmetric broadening of the [001] rod -- mainly to the left side of the SiGe Bragg peak. This sample lies in the regime $\mathrm{w} / \mathrm{d}<0.2$, and the epilayer and its diffraction pattern are essentially bimodal: there are still-perfect domains which produce intensity only on the [001] rod, and distorted domains which produce the diffuse-scattering features. Since in this regime the dislocation displacement fields are to first order not overlapping, the diffuse-scattering features seen here are directly related to the reciprocal space structure of individual dislocations. Assuming the dislocations are randomly spaced so as to be incoherent with one another, the diffuse intensity is simply the sum of intensities produced by each of the dislocations. Thus, the pattern is not expected to change shape as dislocation density changes for the regime $\mathrm{w} / \mathrm{d}<0.2$. Instead, there is a transfer of intensity from "nearly perfect" domains to "more distorted" domains within the boundaries defined by the cutoff width, $w$, about the grid formed by the dislocations.

In Fig. 3(b), the direction of the $\mathrm{SiGe}$ (113) reciprocal lattice vector, and a direction orthogonal to it, which is a symmetry axis for the diffuse pattern, are indicated. This second axis is in a direction in reciprocal space that corresponds to the rocking angle, $\omega$, of the diffractometer in real space. The fact that the diffuse intensity introduced by the dislocations lies exactly along this axis indicates that it arises from a distribution of rotations of (113) planes. These must be small rotations of the (113) planes about the $[1,-1,0]$ dislocation line that result from the displacement fields of dislocations normal to the diffraction plane. The axis connecting the two diffuse disks in Fig. 3(b) remains normal to the diffraction vector for all of the (hkl) diffuse-scattering patterns that we have examined: (004), (224), (335) and (044), as well as (113), reinforcing this interpretation. The present results indicate that the disks do not arise from finite-domainsize or shape effects associated with the displacement fields of the dislocations in the manner described by Kidd et al. [4], who argued only from (004) maps, and were therefore experimentally unaware of the rotational nature of the diffuse intensity associated with dislocations. In addition to examining the diffuse-scattering pattern versus (hkl), we have tomographically examined the (004) diffuse-scattering pattern, by mapping reciprocal space at various azimuthal orientations of the sample, in order to arrive at a complete conceptual picture of the (hkl) reciprocal space structure of a low-density, $60^{\circ}$-misfit-dislocation array in a strained heterolayer. Because of space limitations, we defer detailed discussion of the results to a separate publication.

Fig. 3(c) shows the map of a sample annealed to produce a misfit density of $4700 / \mathrm{cm}$. This sample lies near the threshold of the transitional regime $0.2<w / d<1$, and there is an emerging difference in the shape of the diffuse intensity relative to Fig. 3(b). The change of shape results from the fact that the actual spacing of the array is not very uniform [4]. As the dislocation lines of the array on average become closer and the displacement fields become increasingly threedimensional, an observable fraction of dislocations with the same line direction also begin to randomly overlap due to the nonuniformity in the spacing. Superposition of the displacement fields of closely spaced, parallel dislocations produces the wider range of tilts observed in Fig. 3 (c). Closely inspecting Fig. 3(c), one can perhaps see a poorly defined second set of lobes appearing at the edge of the original disks, with about twice the spacing of the original disks. On the left side of the pattern there is perhaps even a third-order lobe. One speculative interpretation of this pattern is that there are some very closely spaced dislocation bunches in the sample (pairs and triplets in this case) which give rise to integer multiples of the single-dislocation tilt distribution -- again through superposition. Fig. 4(a) shows the map of a sample with a misfit density of $6600 / \mathrm{cm}$-- less than a factor of two increase. Because of the increasing probability of random overlap of parallel dislocations, the pattern continues to evolve in shape, as well as intensity.

Finally, Fig. $4(\mathrm{~b})$ shows a sample annealed to a misfit density of $\approx 18,000 / \mathrm{cm}$ where $w / d \approx 0.8$. The transitional regime is nearing completion; and parallel dislocations are beginning to very strongly overlap giving rise to both a wider range of tilts, and a loss of the distinct disks 


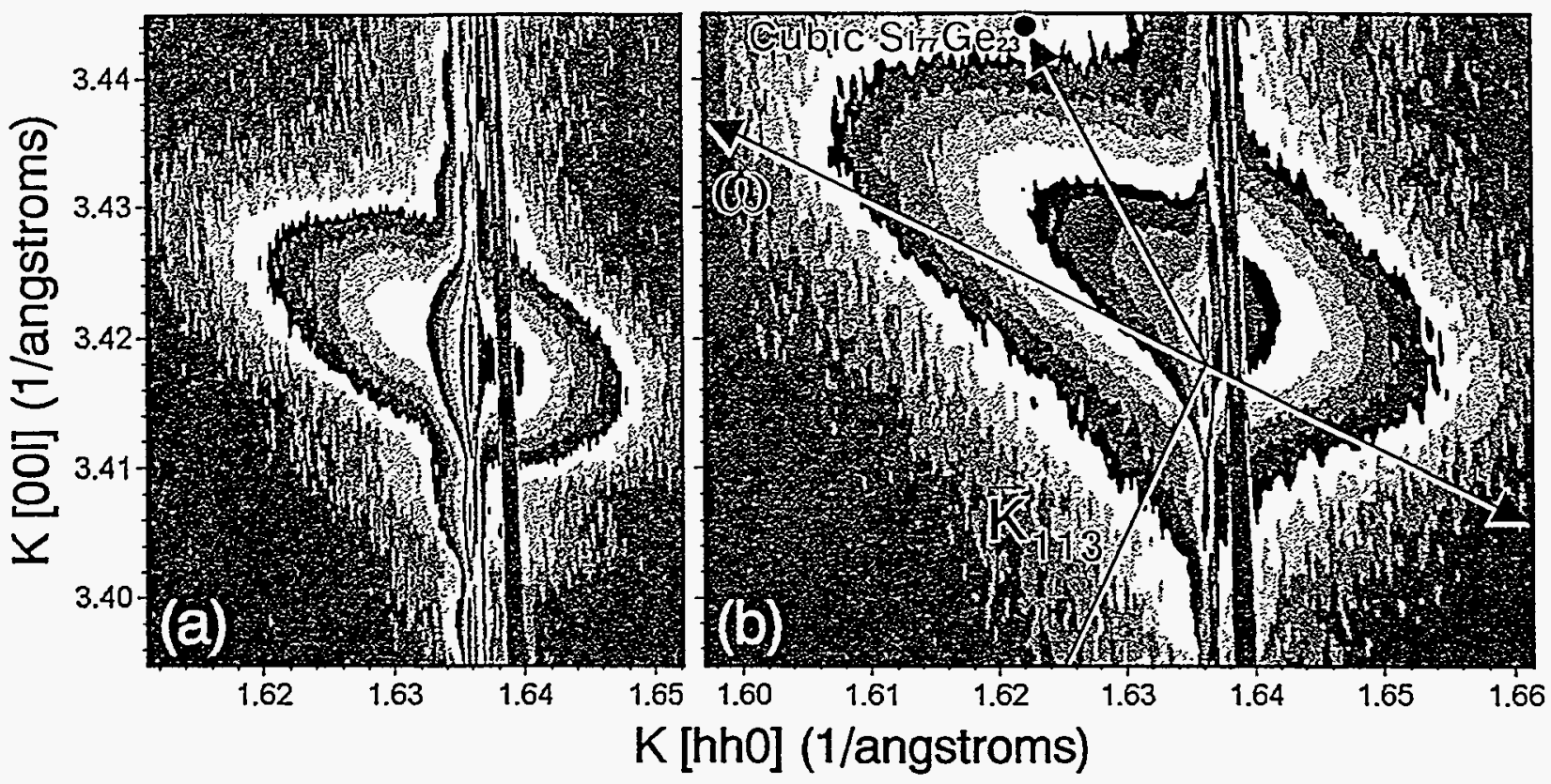

Figure 4. (113) reciprocal space maps of $748 \AA$-thick $\mathrm{Si}_{78} \mathrm{Ge}_{22}$ : (a) annealed at $750{ }^{\circ} \mathrm{C}$ for 1 hour and 45 minutes, and (b) annealed at $775^{\circ} \mathrm{C}$ for 1 hour.

associated with the displacement fields of nonoverlapping, single dislocations. Now, the displacement fields throughout the epilayer are almost fully three-dimensional in character, and new dislocations introduced into the layer fall within the displacement fields of existing dislocations along both $\langle 110\rangle$ line directions. This is the onset of a final stage where the epilayer begins the process of substantial strain relaxation. In addition to continued broadening along the tilt direction, the pattern in Fig. 4(b) now begins to move in the direction in reciprocal space corresponding to long-range, macroscopic strain relaxation of the epilayer.

It is important to note that asymmetric (hhl) diffraction experiments (i.e., our (113) examples) do not directly probe all of the tilts that result from three-dimensional conversion of the epilayer. Dislocations parallel to the diffraction plane produce tilts that are normal to the diffraction plane and therefore cannot be seen. In order to more directly probe the transition to three-dimensional displacement fields, (0kk), or grazing-incidence (h00) maps which simultaneously probe tilts due to both line directions of the array are of potential interest.

\section{MEASUREMENT OF DISLOCATION DENSITY USING DIFFUSE INTENSITY}

Let us consider how the normalized-integrated-diffuse intensity can be used to determine lineal dislocation density. First, we obtained the integrated diffuse intensity, $\mathrm{I}_{\text {diffuse }}$, by summing up the measured diffuse intensity in regions adjacent to the Bragg peak of the SiGe epilayer. This is in contrast to the technique recently used by Goorsky et al. [5], where the diffuse intensity adjacent to the GaAs substrate peak, produced by dislocations in overlying InGaAs heterolayers, was used to determine the dislocation density. Here, the diffuse intensity integration boundary goes to within about $\pm 6 \times 10^{-4} \AA^{-1}$ of the [001] rod and excludes this small region for all [001]. Contributions to the diffuse intensity due to instrumental background effects, such as the energy dispersion adjacent to the SiGe Bragg peak noted above for the as-grown sample of Fig. 3(a), were corrected for by subtracting the background diffuse intensity of dislocation-free reference samples. The diffuse intensity was then normalized by the integrated intensity associated with the entire epilayer, $\mathrm{I}_{\mathrm{epi}}$, which includes both diffuse and Bragg components lying on [001].

Lineal- misfit- dislocation densities determined by etching and counting, versus (113) reciprocal-space measurements of the normalized diffuse intensity, are plotted as symbols in Fig. 5. Results are shown for both 748 and $1208 \AA$-thick films. Using the normalized diffuse intensity to remove the effect of film thickness, the data for these two different-thickness films fall onto a single unified curve. The present experimental results show that dislocation densities from 
400 to 20,000 lines/cm are measurable by the present technique. The lower bound corresponds to a diffuse-intensity signal-tobackground ratio of unity, and the upper bound corresponds to the point where the entire epilayer begins to diffract in the socalled "diffuse" regime, i.e., $\mathrm{I}_{\text {diffuse }} / \mathrm{I}_{\mathrm{epi}}=1$.

The equation for $V_{t}$ in Fig. 1 may be rearranged to obtain the dislocation density, $1 / \mathrm{d}$, given the volume fraction of displaced material, $V_{t}$. We need only connect $V_{t}$ to the diffuse $\mathrm{x}$-ray intensity, and our geometric model will predict dislocation density from the diffuse intensity. In kinematic diffraction the total integrated intensity is linearly proportional to the total number of diffracting atoms assuming absorption is negligible. For

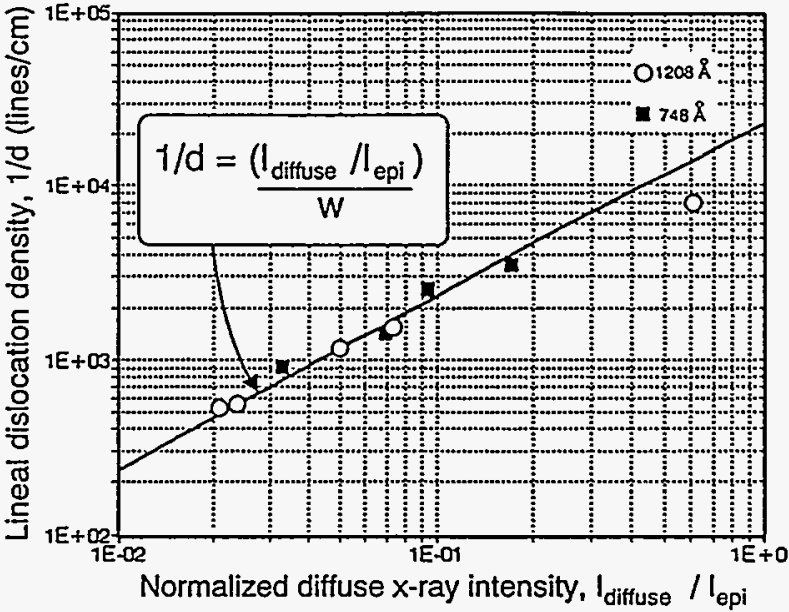

Figure 5. Measured dislocation density versus normalized diffuse $x$-ray intensity. our thin, highly strained SiGe films both dynamical and absorption effects have only a small effect on total intensity. If it is further assumed that displacement field domains diffract essentially independently from perfect domains (we are in essence neglecting interference effects near the integration boundary), we arrive at the general conclusion that the volume fraction of displaced material is approximately the total normalized diffuse $x$-ray intensity.

Now, for the special case of $(\mathrm{hhl})$ diffraction with one $\langle 110\rangle$ direction parallel to the diffraction plane, we can only measure the diffuse intensity of $60^{\circ}$-misfit dislocations normal to the diffraction plane. In this case, we can consider each line direction independently, and the general model simplifies; the volume fraction of displaced material associated with only one $<110>$ line direction is just $\mathrm{V}_{<110\rangle}=\mathrm{w} / \mathrm{d}$; and $\mathrm{V}_{<110\rangle}=\mathrm{I}_{\text {diffuse }(\mathrm{hhl})} / \mathrm{I}_{\text {epi }}$. We arrive at the equation relating misfit density to the normalized (hhl) diffuse intensity shown in Fig. 5. The only free parameter is the effective width of the displacement field, w, which was determined to be $4300 \AA$ by fitting to the experiment. The solid line in Fig. 5 is the fit of the model to the experiment.

The resulting value of $w$ is thought to be directly related to the width of the reciprocal-space boundary near the [001] rod when the integrated diffuse intensity is calculated. Truncation of the displacement field in real space corresponds to the diffuse integration truncation in reciprocal space. The reciprocal-space integration boundary $\pm 6 \times 10^{-4} \AA^{-1}$ from the [001] rod, when interpreted as a tilt, corresponds to a rotation of (113) planes through an angle of $\pm 36^{\prime \prime}$. Calculations of the dislocation displacement fields in these films are being pursued to determine if elasticity theory predicts a similar (113) rotation at $\approx 2150 \AA$ from the dislocation core. The fact that both film thicknesses that were investigated lie on a single curve when the same integration boundary is used, suggests that the displacement fields at the constant real-space cutoff are similar for films of varying thickness. In addition to calculations, additional experiments using a wider range of film thicknesses are planned in order to more thoroughly examine these ideas.

\section{REFERENCES and ACKNOWLEDGMENTS}

We thank Jay Penn for technically assisting the MBE growth of the SiGe films and Mike Moran for assisting with the Nomarski optical microscopy. This work was supported by the U.S. Department of Energy under Contract DE-AC04-94AL85000.

[1] L. R. Thompson, G. J. Collins, B. L. Doyle and J. A. Knapp, J. Appl. Phys. 70, 4760 (1991).

[2] S. T. Picraux, B. L. Doyle and J. Y. Tsao, in Semiconductors and Semimetals 33, edited by T. P. Pearsall (Academic Press, Boston, 1991), pp. 139-222.

[3] S. R. Lee, B. L. Doyle, T. J. Drummond, J. W. Medernach and R. P. Schneider, Jr., in Advances in $X$-Ray Analysis 38, edited by P. Predecki et al. (Plenum Press, New York, 1995), pp. 201-213.

[4] P. Kidd, P. F. Fewster and N. L. Andrew, J. Phys. D 28, Al33 (1995).

[5] M. S. Goorsky, M. Meshkinpour, D. C. Streit and T. R. Block, J. Phys. D 28, A92 (1995). 